\title{
Electro-vortex flow simulation using coupled meshes
}

\author{
Norbert Weber, Pascal Beckstein, Vladimir Galindo, Marco Starace, Tom \\ Weier \\ Helmholtz-Zentrum Dresden - Rossendorf, Dresden, Germany
}

\begin{abstract}
A numerical model for simulating electro-vortical flows in OpenFOAM is developed. Electric potential and current are solved in coupled solid-liquid conductors by a parent-child mesh technique. The magnetic field is computed using a combination of Biot-Savart's law and induction equation. Further, a PCG solver with special regularisation for the electric potential is derived and implemented. Finally, a performance analysis is presented and the solver is validated against several test cases.

Keywords: electro-vortex flow, OpenFOAM, coupled parent child mesh
\end{abstract}

\section{Introduction}

Electro-vortex flow is highly relevant in many industrial processes. Possible applications span from electromagnetic stirring [1] for grain size reduction in solidification [2, 3] over electrode welding [4], electroslag welding, electroslag (re-)melting [5, 6, vacuum arc melting [7] to electrolytic reduction (of e.g. aluminium [8]). Further, many technical devices, as liquid fuses [9], electric jet engines, arc furnaces [10] and liquid metal batteries [11-13] involve or rely on electro-vortex flows. For an overview about such flows, see [14 16].

Electro-vortex flow is not an instability. It develops at (or near) a changing cross-section of a (liquid) conductor. Radial currents produce, together with their own magnetic field, a Lorentz force, which is non-conservative, i.e. its curl is not equal to zero. This force cannot be compensated totally by a pressure 
gradient and therefore drives a flow. For an illustrative example, see Shercliff [17.

Numerical simulation of electro-vortex flow is easy when modelling only the fluid, or a non-conducting obstacle inside a fluid. However, in most realistic cases, electric current passes from solid to liquid conductors and vice versa. The electric potential in these regions must therefore be solved in a coupled way. The classical, segregated approach means solving an equation in each region, and coupling the potential only at the interfaces by suitable boundary conditions [11. While that is easy to implement, convergence is rather poor. An implicit coupling of the different regions by block matrices is a sophisticated alternative for increasing convergence [18. However, it is memory-intensive and by no means easy to implement.

In this article we will present an alternative effective option for region coupling in OpenFOAM. We solve global variables (electric potential, current density) on a global mesh with a variable electric conductivity according to the underlying material. We then map the current density to the fluid regions and compute the electromagnetic induced flow there. This parent-child mesh technique was already used for the similar problem of thermal conduction [19, 20] and just recently for the solution of eddy-current problems with the finite volume method [21].

\section{Mathematical and numerical model}

\subsection{Overview}

The presented multi-region approach is based on a single phase incompressible magnetohydrodynamic (MHD) model [11, 22. The flow in the fluid is described by the Navier-Stokes equation (NSE)

$$
\frac{\partial \boldsymbol{u}}{\partial t}+(\boldsymbol{u} \cdot \nabla) \boldsymbol{u}=-\nabla p+\nu \Delta \boldsymbol{u}+\frac{\boldsymbol{J} \times \boldsymbol{B}}{\rho},
$$

with $\boldsymbol{u}$ denoting the velocity, $t$ the time, $p$ the modified pressure, $\nu$ the kinematic viscosity and $\rho$ the density. The fluid flow is modelled as laminar only; adding 
a turbulence model is planned for the future. We split the electric potential $\phi$, the current density $\boldsymbol{J}$ and the magnetic field $\boldsymbol{B}$ into a constant (subscript 0) and induced part (lower case) as

$$
\begin{gathered}
\phi=\phi_{0}+\varphi \\
\boldsymbol{J}=\boldsymbol{J}_{0}+\boldsymbol{j} \\
\boldsymbol{B}=\boldsymbol{B}_{0}+\boldsymbol{b} .
\end{gathered}
$$

In order to determine the distribution of the constant part of the electric potential $\phi_{0}$ we solve a Laplace equation for the electric potential

$$
\nabla \cdot \sigma \nabla \phi_{0}=0
$$

on the global mesh. The above equation is obtained starting from the Kirchhoff law of charge conservation $\left(\nabla \cdot \boldsymbol{J}_{0}=0\right)$ and $\boldsymbol{J}_{0}=-\sigma \nabla \phi_{0}$. Note that the conductivity $\sigma$ is a field and not a constant, because the equation is solved on the full geometry. During mesh generation, it is ensured that the border between two materials always coincide with a face between two neighbouring cells. The global current density is then calculated as

$$
\boldsymbol{J}_{0}=-\sigma \nabla \phi_{0}
$$

and mapped to the fluid region. Afterwards, the constant magnetic field is determined as described in section 2.1.1 only in the fluid.

Often it is sufficient to calculate only the constant current and magnetic field. Nevertheless, our solver also allows to compute their induced counterparts, e.g. for simulating the Tayler instability [23 31]. The scheme is similar to that described above: in a first step, the induced electric potential $\varphi$ is determined by solving a Poisson equation

$$
\nabla \cdot \sigma \nabla \varphi=\nabla \cdot \sigma(\boldsymbol{u} \times \boldsymbol{B})
$$

after mapping the source term $\boldsymbol{u} \times \boldsymbol{B}$ to the global mesh. The induced current can be computed taking into account Ohm's law

$$
\boldsymbol{j}=\sigma(-\nabla \varphi+\boldsymbol{u} \times \boldsymbol{B}) .
$$




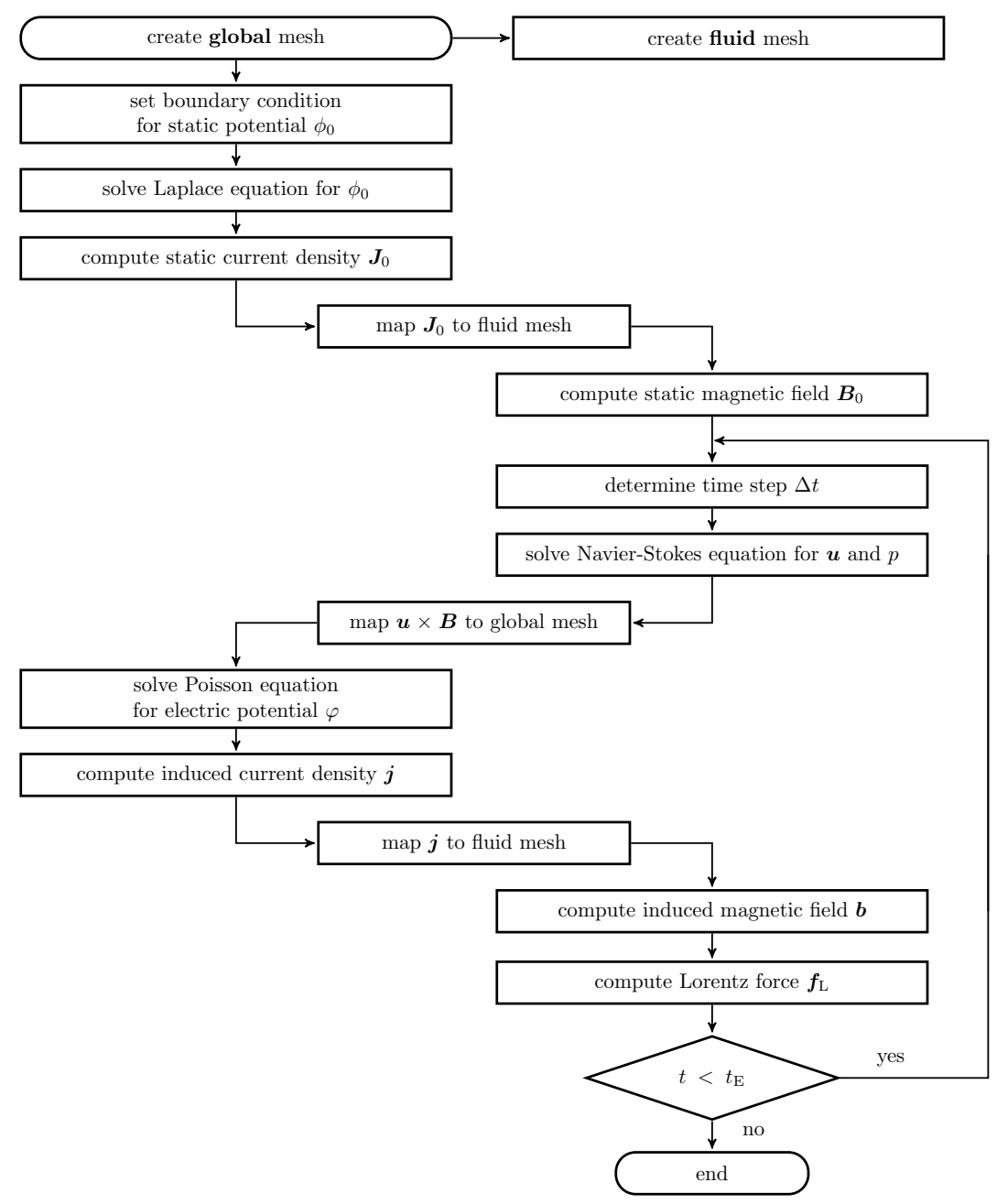

Figure 1: Flowchart of the simulation model.

After mapping $\boldsymbol{j}$ to the fluid mesh we determine the induced magnetic field as described in section 2.1.1.

Our model is not capable of describing AC currents, because we use the quasi-static approximations by neglecting the temporal derivation of the vector potential $(d \boldsymbol{a} / d t=0)$ and magnetic field $(d \boldsymbol{b} / d t=0)$ 32. For a detailed flowchart of the model, please refer to figure 1 . 


\subsubsection{Computation of the magnetic field}

For the computation of both, the constant part of the magnetic field $\boldsymbol{B}_{0}$ and its induced counterpart $\boldsymbol{b}$ we use the inversion of Ampère's law, the Biot-Savart integral

$$
\boldsymbol{B}(\boldsymbol{r})=\frac{\mu_{0}}{4 \pi} \int \frac{\boldsymbol{J}\left(\boldsymbol{r}^{\prime}\right) \times\left(\boldsymbol{r}-\boldsymbol{r}^{\prime}\right)}{\left|\boldsymbol{r}-\boldsymbol{r}^{\prime}\right|^{3}} d V^{\prime}
$$

to determine both from the current density $\boldsymbol{J}$. This integro-differential approach was proposed by Meir and Schmidt [33 38 and later used for describing dynamos [39 41] and the Tayler instability [22].

In order to obtain the magnetic field in one single cell (at the position $\boldsymbol{r}$ ), the electric current densities of all other cells (at the position $\boldsymbol{r}^{\prime}$ ) have to be integrated. The number of operations is therefore equal to the number of cells squared. This way of computation is extremely costly. We will explain here several ways for a speed up of the procedure. Solving Biot-Savart's integral on a coarser grid, recalculating it every nth time step, and an appropriate parallelisation [22] are the most simple ways.

The parallelisation is implemented in OpenFOAM using MPI. Basically, each processor contains only the current density of its local cells. With this, it computes the magnetic field for the full geometry (see figure 2a). Finally, the field $\boldsymbol{B}$ of each cell has to be summed up over all processors. This might be done using the MPI function ALLREDUCE, resulting in a correct and global $\boldsymbol{B}$ on all processors. However, this is not necessary, because a single processor needs only its local $\boldsymbol{B}$ for further computation. Therefore, each processor receives only its local magnetic field from all other processors and adds up all contributions given. The communication process is illustrated in figure $2 \mathrm{~b}$.

Increasing the speed-up considerably is possible by computing Biot-Savart's integral only on the boundaries and solving the induction equations 42, 43.

$$
\begin{aligned}
& 0=\Delta \boldsymbol{B}_{0} \\
& 0=\frac{1}{\sigma \mu_{0}} \Delta \boldsymbol{b}+\nabla \times\left(\boldsymbol{u} \times \boldsymbol{B}_{0}\right)+\nabla \times(\boldsymbol{u} \times \boldsymbol{b})
\end{aligned}
$$

for the constant and induced magnetic field in the quasi-static limit 32 . 

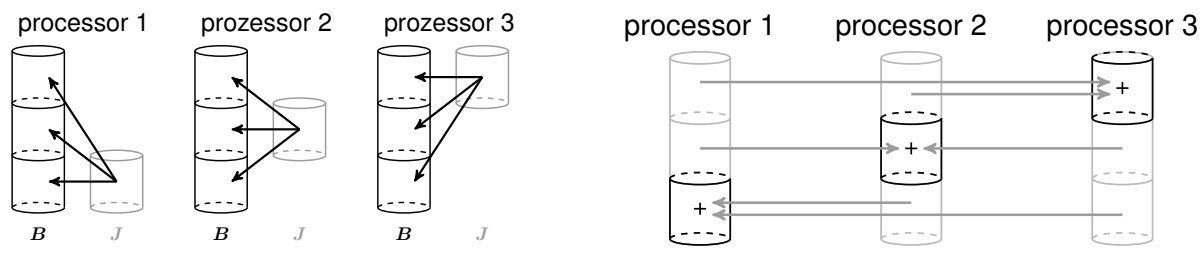

(a)

(b)

Figure 2: Each processor computes a full magnetic field from its local current $\boldsymbol{J}$ (a), receives afterwards only its local $\boldsymbol{B}$ from all other processors and adds it up (b).

An even faster alternative is shifting the problem from the magnetic field $\boldsymbol{B}$ to the vector potential $\boldsymbol{A}$ using the relation $\boldsymbol{B}=\nabla \times \boldsymbol{A}$. Similar to Biot-Savart's law for $\boldsymbol{B}$, the vector potential can be determined by Green's identity [44:

$$
\boldsymbol{A}(\boldsymbol{r})=\frac{\mu_{0}}{4 \pi} \int \frac{\boldsymbol{J}\left(\boldsymbol{r}^{\prime}\right)}{\left|\boldsymbol{r}-\boldsymbol{r}^{\prime}\right|} d V^{\prime} .
$$

Please note that this formula is much cheaper to compute than Biot-Savart's law (equation 9) [45, 46].

The transport equations for the vector potential are derived from Ampère's law, $\boldsymbol{B}=\nabla \times \boldsymbol{A}$, Ohm's law [4] and using the Coulomb gauge condition $\nabla \cdot \boldsymbol{A}=0$ as

$$
\begin{aligned}
& 0=\frac{1}{\sigma \mu_{0}} \Delta \boldsymbol{A}_{0}-\nabla \phi_{0} \\
& 0=\frac{1}{\sigma \mu_{0}} \Delta \boldsymbol{a}+\boldsymbol{u} \times \boldsymbol{B}_{0}+\boldsymbol{u} \times(\nabla \times \boldsymbol{a})-\nabla \varphi .
\end{aligned}
$$

Basically all mentioned approaches of determining $\boldsymbol{B}$ based on the equations (9) till (14) are equal from a physical point of view. But due to the way they are discretised and numerically solved, there will be differences in both accuracy and calculation time. While being the most expensive method, calculating the magnetic field by means of Biot-Savart's law also gives the most accurate result. This stems from the fact that the integral equation 9 represents an exact solution for $\boldsymbol{B}$ which is only numerically integrated for a finite number of cells. As already mentioned, a computationally less expensive evaluation can be achieved with the help of the magnetic vector potential $\boldsymbol{A}$ and Green's identity 
(12), where the complexity of the integrand is reduced compared to equation (9). Despite of equation (12) also being an exact solution, the subsequent calculation of $\boldsymbol{B}=\nabla \times \boldsymbol{A}$ introduces an additional layer of discretisation errors from cell averaging and face interpolation.

As outlined above, Biot-Savart's law may be used also in combination with equations (10) and 11) or Green's identity 12 combined with equations 13 . and (14), while only boundary values of $\boldsymbol{B}$ or $\boldsymbol{A}$ are evaluated using the exact integral equations. Internal values are then recovered from solving the related differential equations. This drastically improves computational efficiency at the cost of some accuracy. However, from figure 1 one can comprehend that it is sufficient to calculate $\boldsymbol{B}_{0}$ or $\boldsymbol{A}_{0}$ only once at the beginning of a simulation, whereas $\boldsymbol{b}$ or $\boldsymbol{a}$ needs to be updated recurringly while marching in time. The most promising way of determining the total magnetic field $\boldsymbol{B}$ is thus to compute its static part $\boldsymbol{B}_{0}$ once and solely using Biot-Savart's law with the current density $\boldsymbol{J}_{0}$ and the induced part $\boldsymbol{b}$ mediately from $\boldsymbol{b}=\nabla \times \boldsymbol{a}$, whereby the solution of the induced magnetic vector potential $\boldsymbol{a}$ is in turn based on the transport equation (14) for which Dirichlet boundary conditions are derived from evaluating Green's identity with the current density $j$. This approach has been used for all following calculations.

In this way, $\boldsymbol{B}_{0}$ is most accurate and $\boldsymbol{b}$ is repeatedly calculated with minimum computational effort. Another important advantage of this realisation is that the solenoidal nature of $\boldsymbol{B}$ is implicitly satisfied, as $\boldsymbol{B}_{0}$ results from an exact solution in shape of Biot-Savart's law and $\boldsymbol{b}$ is calculated from the definition of the induced vector potential $\boldsymbol{a}$ with $\nabla \cdot \boldsymbol{b}=\nabla \cdot(\nabla \times \boldsymbol{a}) \equiv 0$. Numerically, Gauss's law $\nabla \cdot \boldsymbol{B}=0$ is of course only met approximatively due to discretisation errors. With linear interpolation, the corresponding finite volume approximation is second order accurate. Additionally, for small magnetic Reynolds numbers, which are typical for most liquid metal MHD flows on laboratory scale, $\boldsymbol{b}$ is usually small compared to $\boldsymbol{B}_{0}$.

As opposed to using $\boldsymbol{b}=\nabla \times \boldsymbol{a}$, a direct solution of the induction equation 111) for $\boldsymbol{b}$ would in general require additional steps to ensure its solenoidal 
property. This is particularly true in ideal or plasma MHD for time-dependent problems at high magnetic Reynolds numbers, where the induction equation is dominated by convection 48. For such cases it is usually necessary to adopt a special correction. An overview of possible divergence cleaning methods can be found in [49]. The OpenFOAM standard solver mhdFoam for example uses the projection method, which is well known from the pressure-velocity coupling of the Navier-Stokes equations.

Accordingly, also the solution of equation (14) would generally require some correction to maintain the solenoidal property of the magnetic vector potential $\boldsymbol{a}$. However $\nabla \cdot \boldsymbol{b}=0$ is a physical requirement, whereas $\nabla \cdot \boldsymbol{a}=0$ is just a gauge. If the Helmholtz decomposition $\boldsymbol{a}=\nabla \times \boldsymbol{\Pi}+\nabla \Psi$ is consulted, it can be easily seen that $\nabla \cdot \boldsymbol{a}=\nabla \cdot \nabla \Psi$ does not influence the magnetic field $\boldsymbol{b}=\nabla \times \boldsymbol{a}=\nabla \times \nabla \times \boldsymbol{\Pi}$. Thus, even a weakly satisfied Coulomb gauge $\nabla \cdot \boldsymbol{a} \approx 0$ would suffice to correctly represent $\boldsymbol{b}$. Fixing the gauge is merely important to achieve a unique solution for $\boldsymbol{a}$. Moreover, for diffusively dominated cases the Coulomb gauge may be incorporated directly into Ampère's law according to $\nabla \times \nabla \times \boldsymbol{a}-\nabla(\nabla \cdot \boldsymbol{a})=-\nabla \cdot \nabla \boldsymbol{a}=\mu_{0} \boldsymbol{j}$ as long as charge conservation $\nabla \cdot \boldsymbol{j}=0$ is satisfied [50]. This requirement is met with the solution of 7) In equation (14), the sum $\boldsymbol{u} \times \boldsymbol{B}_{0}+\boldsymbol{u} \times(\nabla \times \boldsymbol{a})-\nabla \varphi$ corresponds to the induced current

density $\boldsymbol{j} / \sigma$ (8). If we explicitly discretise these terms, they can be regarded as one source term for a Poisson equation, whose system matrix is symmetric. For cases like this, it was demonstrated in [21] that indeed no additional divergence cleaning for $\boldsymbol{a}$ is required.

\section{Discretisation}

Special attention must be paid to the discretisation of the Laplace term $\nabla \cdot(\sigma \nabla \phi)$ of equation (5) and (7) because of the sharp jump in conductivity between different materials. This jump is not smeared, but exactly reproduced in our model. A linear interpolation of $\sigma$ would lead to a wrong potential near the interface. 
For a consistent application of the Gauss theorem to discretise the equations (see [51]), the electric conductivity is interpolated harmonically. Knowing that the potential $\phi_{f}$ and the normal current $(\boldsymbol{j} \cdot \boldsymbol{n})_{f}$ must be continous from a cell $P$ to its neighbour $N$, we find the conductivity at the face $f$ to be

$$
\sigma_{f}=\left(\frac{\left(\delta_{P} / \delta\right)}{\sigma_{P}}+\frac{\left(\delta_{N} / \delta\right)}{\sigma_{N}}\right)^{-1}
$$

with $\delta_{i}$ denoting the distance cell centre - face and $\delta$ the distance between both cell centres. In the quasi-static limit, this exactly matches the embedded discretisation scheme which was derived in 21] to get a proper discretisation of the Laplacian. For a more detailed discussion and similar discretisation of the thermal conductivity, see [52 55].

Secondly, care must be taken when computing the gradient of the potential to determine the current density as $\boldsymbol{J}=-\sigma \nabla \phi$. In order to be able to use the Gauss theorem for discretisation, the electric potential on the faces must be determined. Using the same assumptions as for the harmonic interpolation described above, we identify the electric potential at the face as

$$
\phi_{f}=w \phi_{P}+(1-w) \phi_{N}
$$

with the interpolation weight

$$
w=\frac{\delta_{N} \sigma_{P}}{\delta_{P} \sigma_{N}+\delta_{N} \sigma_{P}} .
$$

As before, this interpolation scheme corresponds to the embedded discretisation of the gradient from [21] in case of the quasi-static assumption. All other discretisation schemes do not need special attention. We use backward differencing for time discretisation, a mixed linear-upwind scheme for the convective term in equation (1) and second order linear discretisation for all other schemes.

\section{Equation solvers}

The solution procedure of our model is illustrated in figure 1. As the NavierStokes equation is discretised and solved by means of the PISO-algorithm [56], 
four different Poisson equations need to be addressed. This comprises the Laplace equation for the static potential $\phi_{0}$, one Poisson equation for the vector potential $\boldsymbol{a}$, one Poisson equation for the potential $\varphi$ and another Poisson equation for the fluid pressure $p$. Especially the latter two are most commonly solved for Neumann boundary conditions. To improve the overall robustness of the solution process in connection with the employed parent-child mesh approach, we have implemented an alternative regularisation technique for the iterative equation solvers in OpenFOAM, which is briefly explained in the following.

The discretisation of a Poisson equation leads to a linear equation system

$$
\mathbf{M} \psi=\mathbf{r}
$$

where $\mathbf{M} \in \mathbb{R}^{n \times n}$ is a symmetric positive semi-definite matrix, $\boldsymbol{\psi} \in \mathbb{R}^{n}$ is the discrete solution vector for either $\varphi$ or $p$, and the right-hand side $\mathbf{r} \in \mathbb{R}^{n}$ mainly represents the inhomogeneous part. Each row of the system $\sqrt{18}$ is related to one of $n$ cells. In case of a Neumann problem, the system matrix will be singular and the solution is only defined up to an additive constant vector. More specifically, the one-vector $\mathbf{1}=(1,1, \ldots, 1,1)^{T}$ lies in the null space of the linear map $\mathbf{M} \boldsymbol{\psi}$. In other words, $\mathbf{v}_{1}=1 / \sqrt{n}$ is a normalized eigenvector corresponding to the eigenvalue $\lambda_{1}=0$ in accordance with the identity $\left(\mathbf{M}-\lambda_{1} \mathbf{I}\right) \mathbf{v}_{1}=\mathbf{0}$.

In OpenFOAM such a singular matrix $\mathbf{M}$ is regularised by means of adding the equation

$$
\mathrm{c}_{R} \psi_{P}=\mathrm{c}_{R} \psi_{R}
$$

to the row which belongs to cell $P$, where $c_{R}$ is initially an arbitrary coefficient, $\psi_{P}$ is the unknown solution and $\psi_{R}$ is a reference solution for that cell. In order to slightly increase diagonal dominance of $\mathbf{M}, \mathbf{c}_{R}$ is usually set to the diagonal coefficient of the matrix before adding the equation: $\mathrm{c}_{R}=\mathrm{m}_{P}$. By specifying the reference value $\psi_{R}$, the solution gets locally constrained in a weak sense. This approach is however extremely sensitive to the smallest errors in the corresponding compatibility condition of the Neumann problem. Such numerical errors may arise from the data exchange between child and parent mesh due to interpolation. 
A much more robust regularisation can be achieved by inverting the idea of the so called Hotelling deflation [57, which is actually a simple technique to solve eigenproblems by selectively shifting single known eigenvalues of a matrix to zero. Conversely, we may use the same procedure to shift them also from zero to an arbitrary value, thus inflating the matrix.

According to the spectral theorem for symmetric matrices [58, it is possible to decompose $\mathbf{M}$ based on its eigenvalues $\lambda_{k}$ and orthonormal eigenvectors $\mathbf{v}_{k}$ :

$$
\mathbf{M}=\sum_{k=1}^{n} \lambda_{k} \mathbf{v}_{k} \mathbf{v}_{k}^{T}=\lambda_{1} \mathbf{v}_{1} \mathbf{v}_{1}^{T}+\sum_{k=2}^{n} \lambda_{k} \mathbf{v}_{k} \mathbf{v}_{k}^{T} .
$$

Using this decomposition we may then create a non-singular matrix $\widetilde{\mathbf{M}}$ using only $\mathbf{v}_{1}$ from above:

$$
\widetilde{\mathbf{M}}=\mathbf{M}+\widetilde{\lambda}_{1} \mathbf{v}_{1} \mathbf{v}_{1}^{T}=\mathbf{M}+\widetilde{\lambda}_{1} \frac{1}{n} \mathbf{1 1}^{T}
$$

where $\widetilde{\lambda}_{1}$ is any non-zero eigenvalue replacing $\lambda_{1}$. It is important to note that $\widetilde{\mathbf{M}}$ does not preserve the original sparsity pattern of $\mathbf{M}$, which is usually undesired. Hence, a direct manipulation would not only mean a waste of memory, but also a contraction in terms of the face addressing of OpenFOAM. However, we may include the modification indirectly when computing the matrix-vector product:

$$
\widetilde{\mathbf{M}} \boldsymbol{\psi}=\mathbf{M} \boldsymbol{\psi}+\widetilde{\lambda}_{1} \frac{1}{n} \mathbf{1 1}^{T} \boldsymbol{\psi}=\mathbf{M} \boldsymbol{\psi}+\widetilde{\lambda}_{1} \frac{1}{n} \sum_{k=1}^{n} \boldsymbol{\psi}_{k} \mathbf{1},
$$

which is essentially the kernel of any iterative equation solver [59]. Furthermore parallelisation is straight-forward as the exchange of the rightmost sum does only require little communication.

Taking the properties of $\mathbf{M}$ into consideration, it can be shown that all of its eigenvalues are smaller or equal to twice the maximum of its diagonal coefficients. Therefore we use the diagonal mean as modified eigenvalue $\widetilde{\lambda}_{1}=\left\langle\mathrm{m}_{P}\right\rangle$, thus preserving the spectral radius of $\mathbf{M}$. Tests with the preconditioned CGmethod [59] showed that the smoothness of the numerical solution is preserved even if errors in the compatibility condition exist. It is exactly this preservation of smoothness which distinguishes our method from the original regularisation technique in OpenFOAM, and which makes our method superior. 


\section{Results}

\subsection{Test case 1: speed-up of Biot-Savart's law}

In this section we present a performance analysis of the magnetic field computation in a cylindrical geometry with an imposed current density $\boldsymbol{J}$ (the other parts of the solver are switched off). The speedup and scaling analysis is carried out on a cluster with Intel 8-Core Xeon 3,3 GHz CPUs cross linked with $40 \mathrm{Gbit} / \mathrm{s}$ Infiniband. The solvers are compiled with OpenFOAM 2.2.0 and MPI 1.6.3.

In a first step we solve only Biot-Savart's law (equation 9) for all cells and boundary faces - on a changing number of processors. The test case contains 352000 cells. Figure 3a shows a good scaling up to 64 processors. The com-
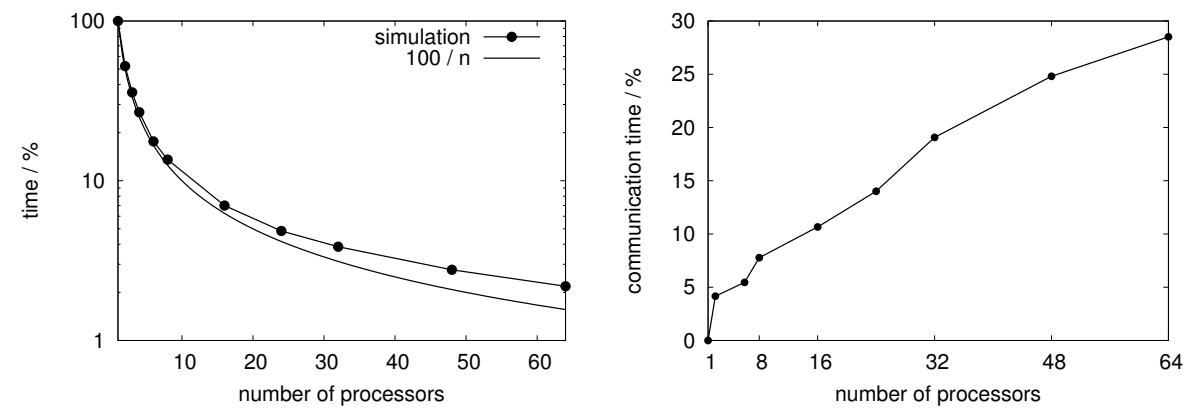

Figure 3: Computation time of Biot-Savart's law on 1 to 64 processors (a) and communication time divided by total time (b).

munication time is $28 \%$ when using all 64 processors. In that case a single processor contains only 5500 cells.

In a second test case, we use the same configuration again and compare the full Biot-Savart integral with the method of solving the induction equation (10). For the latter, we compute Biot-Savart's law only on the patches in order to obtain the correct boundary conditions. Figure 4 shows the relative computation times (total cpu time/(cpu time for simulation in one processor).100\%) for one to 16 processors. The method of using the Biot-Savart law on the boundary regions only together with the solution of the corresponding induction equation 


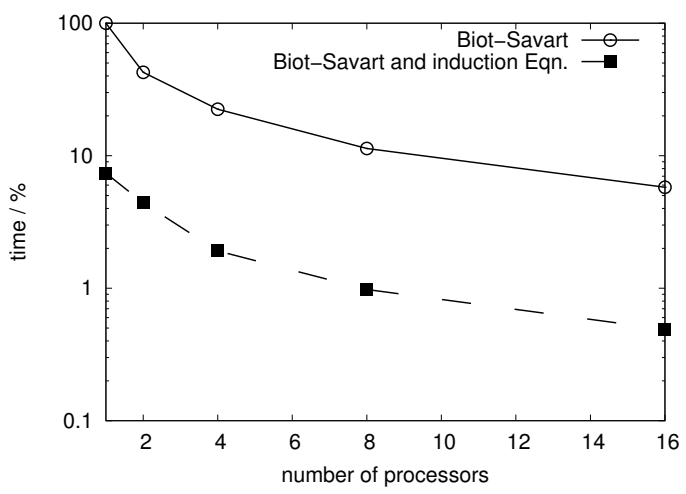

Figure 4: Relative calculation time for the volume based Biot-Savart and the surface based Biot-Savart combined with solving the induction equation.

in the inner region scales very well, too; it is approximately 13.5 times faster than the volume Biot-Savart method. Note that this factor will probably increase for larger problems with more cells.

In a third case we use a mesh with 63200 cells and compare the magnetic field with the vector potential approach. In both cases we firstly compute the boundary conditions and solve then a transport equation for $\boldsymbol{A}$ or $\boldsymbol{B}$ on a single processor 50 times. The fastest result we obtain by using Biot-Savart for the vector potential (equation 12 and 13 p. Computing the magnetic field on the boundary and solving the induction equation (9) and 10$)$ is five times slower. The volume-based Biot-Savart is 84 times slower. Of course this holds only for the Biot-Savart calculation; the differences for the whole solver, where the flow simulation is included, will be smaller.

\subsection{Test case 2: current distribution in $2 D$}

In a second test case the discretisation schemes for electric conductivity and potential are validated by comparison with the commercial software Opera. We simulate a simple two-dimensional geometry $(1 \times 2 \times 0.1 \mathrm{~m})$, consisting of two conductors of very different conductivity with an inclined surface (inclination $45^{\circ}$ ) - see figure $5 \mathrm{a}$. A vertical current of $1 \mathrm{~A}$ is applied. Figure $5 \mathrm{p}$ shows the equipotential lines, figure 5 the current lines and $5 \mathrm{~d}$ the disturbed current. As 
expected, the current lines concentrate in the area of high conductivity. Figure

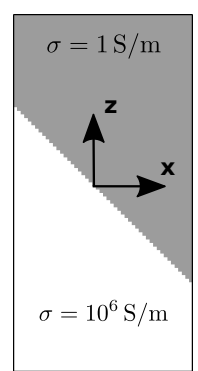

(a)

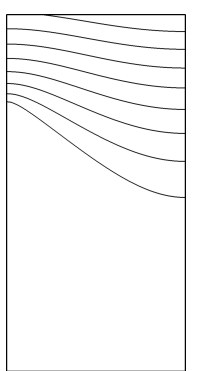

(b)

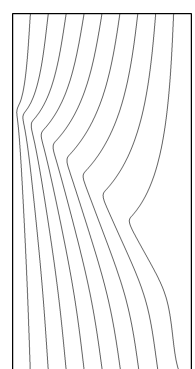

(c)

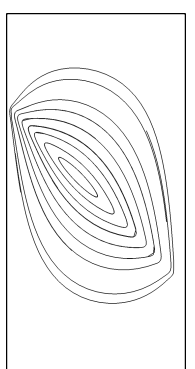

(d)

Figure 5: Conductivity distribution (a), electric potential (b), complete current density (c) and disturbed current density (d). The applied electrical current of $1 \mathrm{~A}$ if flowing upwards.

6 shows the electric potential and the currents along a vertical and horizontal centred line. The result of OpenFOAM and Opera match very well. Obviously, Opera uses Dirichlet boundary conditions for the electric potential (i.e. an equipotential surface) - so the same was done in OpenFOAM.

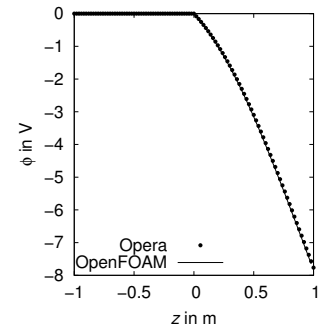

(a)

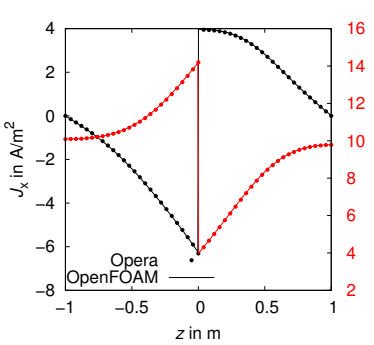

(b)

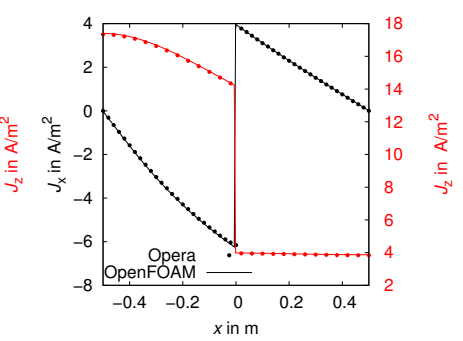

(c)

Figure 6: Electric potential (a) and horizontal current (b) along a centered vertical line for an applied current of $1 \mathrm{~A}$ and current density along a horizontal line at $z=0$.

\subsection{Test case 3: electro-vortex flow in a cylindrical geometry}

Several model experiments [60 62] and similar analytical solutions [63, 64] of electro-vortex flow are known from literature with most of them unfortunately lacking detailed information. Here we will study the well reviewed example of a 
thin electrode touching a cylindrical bath of liquid metal [65]67]. The experiment was conducted at the Institute of Physics in Riga and published by Zhilin et al. 68. Figure 7 illustrates the setup: a horizontal current passes through a cylindrical bath of liquid mercury (colored in blue). One copper electrode covers the whole surface, the other is reduced to a small rod. The whole experiment is embedded into a steel pipe; two mercury filled "buffer zones provide for a smooth current transition" between external wires and the experiment. The axial velocity along the cylinder axis is measured with a spacing of $1 \mathrm{~mm}$ in $x$-direction at $y=0$. The current is increased to up to $1500 \mathrm{~A}$.

Unfortunately, the article does not provide any details about the external current leads. They are therefore assumed to be infinitely long. The measurements colored in red (fig. 7) were not quoted by Zhilin et al. [68, but estimated from the sketch. Similarly, the material properties were not given; they may vary considerably depending on the exact material/alloy. We assume the copper conductivity to be $\sigma_{\mathrm{Cu}}=58.5 \cdot 10^{6} \mathrm{~S} / \mathrm{m}$, the conductivity of mercury as $\sigma_{\mathrm{Hg}}=1.04 \cdot 10^{6} \mathrm{~S} / \mathrm{m}$, its density as $\rho_{\mathrm{Hg}}=13534 \mathrm{~kg} / \mathrm{m}^{3}$ and its kinematic viscosity as $\nu=1.2 \cdot 10^{-7} \mathrm{~m}^{2} / \mathrm{s}[69,70]$. The tube is made of "stainless steel"; we assume therefore an electric conductivity of $\sigma_{\mathrm{St}}=1.4 \cdot 10^{6} \mathrm{~S} / \mathrm{m}$ which is typical for X5CrNi18-10. The tube works as potential divider - only a part of the current passes through the mercury/copper.

Figure 8 8 shows the general flow structure (grid resolution in $\mathrm{Hg} 0.5 \mathrm{~mm}$ ). Assuming infinitely long lateral current leads and neglecting external magnetic

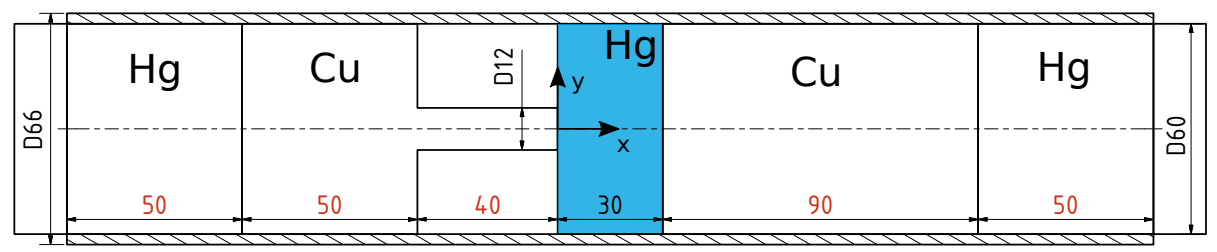

Figure 7: Sketch of the experiment of Zhilin et al. 68. The experiment is modelled with thick lateral current collectors which are $3 \mathrm{~m}$ long (but not shown in the image). The red dimensions (in $\mathrm{mm}$ ) are estimated. The working section (blue) with the symmetry axis $x$ is filled with liquid mercury. 


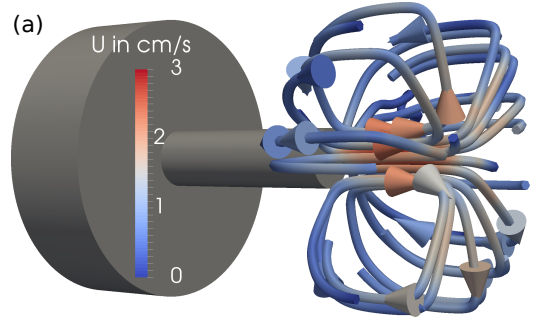

(c)

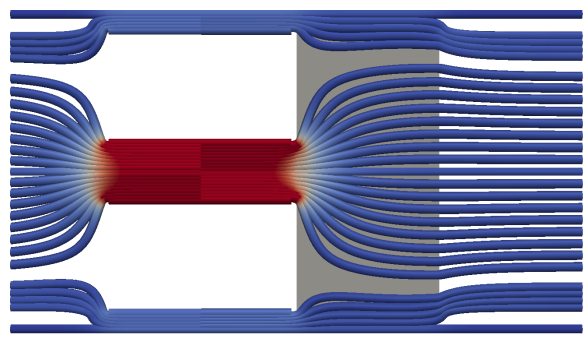

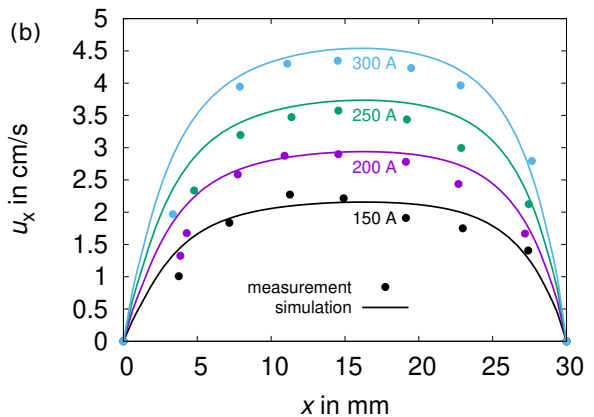

$A / m^{2}$

${ }^{\mathrm{A}}=\mathrm{m}^{2} \mathrm{l} .0 \mathrm{e}+06$

$7.5 e+5$

$5 e+5$

$2.51 e+5$

$1.0 \mathrm{e}+03$ (d)

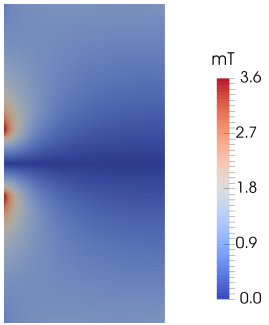

Figure 8: Electro-vortex flow at $I=200 \mathrm{~A}$ (a) and measured axial velocity at the cylinder axis as given by Zhilin et al. [68] (b) as well as the corresponding current density (c) and the magnetic field (d).

fields, we expect exactly such a symmetric flow. Further we expect the velocity along the cylinder axis to be approximately uniform in the middle of the test section (see fig. 8b) as long as the current is not extremely low. The simulated curves for $I=150 \ldots 300$ A fit very well to the measured velocity values (dotted). A certain deviation can be explained by the many unknown experimental parameters; especially the length of the rod has a certain influence on the magnitude of the flow (for details, see Appendix A).

\section{Summary and outlook}

We have developed a solver for electro-vortical flow, using a mesh mapping method. Arbitrary solid and fluid conductors are fully coupled. Electric potential and current density are solved on a global mesh, and copied to the fluid mesh. This parent-child mesh technique is much faster than the classical segregated approach. An improved regularisation technique for the solution of the 
Poisson equation of the electric potential is presented. The magnetic field is computed fully parallelly using Biot-Savart's law. This was shown to be efficient at least up to 64 processors. Calculating Biot-Savart's law only on the boundaries and solving a corresponding induction equation in the fluid region speeds up the magnetic field computation drastically. A first validation of the solver was done using the commercial software Opera and by comparison with experimental data.

The solver presented can easily cope with up to 1 million cells. For larger simulations, a multigrid method or a coarser grid for the magnetic field computation might be necessary. Further, the solver shall be enhanced by a turbulence model and will be further validated using recent experimental data. For a meaningful comparision to experimental data, all dimensions of the setup and all conductivities of the conductors as well the placement of the feeding lines and possible magnetic background fields must be known. Only in that case a computation of the experimentally investigated case can successfully be performed. We aim to use the solver to study electro-vortex flow in liquid metal batteries [13, 71, 72] and aluminium reduction cells 73 , as well as for related experiments [74, 75]. In the long term, a comparison between a segregated, a block matrice coupled (not existing in OpenFOAM yet) and the here presented electro-vortex flow solver is planned.

\section{Acknowledgements}

This work was supported by Helmholtz-Gemeinschaft Deutscher Forschungszentren (HGF) in frame of the Helmholtz Alliance "Liquid metal technologies" (LIMTECH). The computations were performed on the Bull HPC-Cluster "Taurus" at the Center for Information Services and High Performance Computing (ZIH) at TU Dresden and on the cluster "Hydra" at Helmholtz-Zentrum Dresden - Rossendorf. Fruitful discussions with R. Ashour, S. Beale, V. Bojarevics, D. Kelley, A. Kharicha, J. Priede and F. Stefani on several aspects of electrovortex flow are gratefully acknowledged. N. Weber thanks Henrik Schulz for the 
HPC support.

\section{References}

\section{References}

[1] A. Ludwig, M. Wu, A. Kharicha, Simulation in Metallurgical Processing: Recent Developments and Future Perspectives, J. Miner. Met. Mater. Soc. 68 (8) (2016) 2191-2197. doi:10.1007/s11837-016-1992-0.

[2] D. Räbiger, Y. Zhang, V. Galindo, S. Franke, B. Willers, S. Eckert, The relevance of melt convection to grain refinement in AlSi alloys solidified under the impact of electric currents, Acta Mater. 79 (2014) 327-338. doi: $10.1016 / \mathrm{j}$.actamat.2014.07.037.

[3] S. Franke, D. Räbiger, V. Galindo, Y. Zhang, S. Eckert, Investigations of electrically driven liquid metal flows using an ultrasound Doppler flow mapping system, Flow Meas. Instrum. 48 (2016) 64-73. doi:10.1016/j. flowmeasinst.2015.09.004

[4] O. V. Kazak, A. N. Semko, Numerical modeling of electro-vortical flows in a confined volume, J. Eng. Phys. Thermophys. 85 (2012) 1167-1178.

[5] A. Kharicha, W. Schützenhöfer, A. Ludwig, R. Tanzer, M. Wu, On the importance of electric currents flowing directly into the mould during an ESR process, Steel Res. Int. 79 (8) (2008) 632-636.

[6] A. Kharicha, A. Ludwig, M. Wu, On Melting of Electrodes during Electro-Slag Remelting, ISIJ Int. 54 (7) (2014) 1621-1628. doi:10.2355/ isijinternational.54.1621.

[7] A. Kharicha, I. Teplyakov, Y. Ivochkin, M. Wu, A. Ludwig, A. Guseva, Experimental and numerical analysis of free surface deformation in an electrically driven flow, Exp. Therm. Fluid Sci. 62 (2015) 192-201. doi:10.1016/j.expthermflusci.2014.11.014. 
[8] H. Zhang, J. Li, Z. Wang, Y. Xu, Y. Lai, The numerical modeling of melt flow and mhd instabilities in an aluminum reduction cell, J. Miner. Met. Mater. Soc. 11 (2010) 26-31.

[9] A. Cramer, G. Gerbeth, P. Terhoeven, A. Krätschmar, Fluid velocity measurements in electro-vortical flows, Mater. Manuf. Process. 19 (4) (2004) 665-678.

[10] O. Kazak, Modeling of Vortex Flows in Direct Current (DC) Electric Arc Furnace with Different Bottom Electrode Positions, Metall. Mater. Trans. B 44 (5) (2013) 1243-1250. doi:10.1007/s11663-013-9899-4.

[11] N. Weber, V. Galindo, J. Priede, F. Stefani, T. Weier, The influence of current collectors on Tayler instability and electro vortex flows in liquid metal batteries, Phys. Fluids 27 (2015) 014103.

[12] F. Stefani, V. Galindo, C. Kasprzyk, S. Landgraf, M. Seilmayer, M. Starace, N. Weber, T. Weier, Magnetohydrodynamic effects in liquid metal batteries, IOP Conf. Ser. Mater. Sci. Eng. 143 (2016) 012024. doi:10.1088/ 1757-899X/143/1/012024.

[13] T. Weier, A. Bund, W. El-Mofid, G. M. Horstmann, C.-C. Lalau, S. Landgraf, M. Nimtz, M. Starace, F. Stefani, N. Weber, Liquid metal batteries - materials selection and fluid dynamics, IOP Conf. Ser. Mater. Sci. Eng. 228 (2017) 012013.

[14] V. Bojarevičs, Y. Freibergs, E. I. Shilova, E. V. Shcherbinin, Electrically Induced Vortical Flows, Kluwer Academic Publishers, 1989.

[15] P. A. Davidson, An Introduction to Magnetohydrodynamics, Cambridge texts in applied mathematics, Cambridge University Press, 2001.

[16] A. Y. Chudnovskii, Modeling electrovortex flows, Magnetohydrodynamics 25 (3) (1989) 337-341. 
[17] J. A. Shercliff, Fluid motions due to an electric current source, J. Fluid Mech. 40 (1970) 241-250.

[18] H. Rusche, H. Jasak, Implicit solution techniques for coupled multi-field problems Block Solution, Coupled Matrices (2010).

[19] S. B. Beale, H. K. Roth, A. Le, D. H. Jeon, Development of an open source software library for solid oxide fuel cells (2013).

[20] S. B. Beale, H.-W. Choi, J. G. Pharoah, H. K. Roth, H. Jasak, D. H. Jeon, Open-source computational model of a solid oxide fuel cell, Comput. Phys. Commun. 200 (2016) 15-26. doi:10.1016/j.cpc.2015.10.007.

[21] P. Beckstein, V. Galindo, V. Vukčević, Efficient solution of 3D electromagnetic eddy-current problems within the finite volume framework of OpenFOAM, J. Comput. Phys. 344 (2017) 623-646. doi:10.1016/j.jcp. 2017. 05.005 .

[22] N. Weber, V. Galindo, F. Stefani, T. Weier, T. Wondrak, Numerical simulation of the Tayler instability in liquid metals, New J. Phys. 15 (2013) 043034 .

[23] Y. V. Vandakurov, Theory for the stability of a star with a toroidal magnetic field, Astron Zh 49 (2) (1972) 324-333.

[24] R. J. Tayler, The adiabatic stability of stars containing magnetic fields, Mon R Astr Soc 161 (1973) 365-380.

[25] F. Stefani, T. Weier, T. Gundrum, G. Gerbeth, How to circumvent the size limitation of liquid metal batteries due to the Tayler instability, Energy Convers. Manag. 52 (2011) 2982-2986.

[26] M. Seilmayer, F. Stefani, T. Gundrum, T. Weier, G. Gerbeth, M. Gellert, G. Rüdiger, Experimental Evidence for a Transient Tayler Instability in a Cylindrical Liquid-Metal Column, Phys. Rev. Lett. 108 (2012) 244501. 
[27] A. Bonanno, A. Brandenburg, F. D. Sordo, D. Mitra, Breakdown of chiral symmetry during saturation of the Tayler instability, Phys. Rev. E 86 (2012) 016313.

[28] G. Rüdiger, R. Hollerbach, L. L. Kitchatinov, Magnetic Processes in Astrophysics, Wiley-VCH, 2013.

[29] N. Weber, V. Galindo, F. Stefani, T. Weier, Current-driven flow instabilities in large-scale liquid metal batteries, and how to tame them, J. Power Sources 265 (2014) 166-173. doi:10.1016/j.jpowsour.2014.03.055

[30] W. Herreman, C. Nore, L. Cappanera, J.-L. Guermond, Tayler instability in liquid metal columns and liquid metal batteries, J. Fluid Mech. 771 (2015) 79-114. doi:10.1017/jfm.2015.159

[31] F. Stefani, A. Giesecke, N. Weber, T. Weier, On the Synchronizability of Tayler-Spruit and Babcock-Leighton Type Dynamos, Solar Physics 293 (2018) 12. doi:10.1007/s11207-017-1232-y.

[32] V. Bandaru, T. Boeck, D. Krasnov, J. Schumacher, A hybrid finite differenceboundary element procedure for the simulation of turbulent MHD duct flow at finite magnetic Reynolds number, J. Comput. Phys. 304 (2016) 320-339.

[33] A. J. Meir, P. G. Schmidt, A velocity-current formulation for stationary MHD flow, Appl. Math. Comput. 65 (1994) 95-109.

[34] A. J. Meir, P. G. Schmidt, Variational methods for stationary MHD flow under natural interface conditions, Nonlinear Anal. Theory Methods Appl. 26 (1996) 659-689.

[35] P. G. Schmidt, A Galerkin method for time-dependent MHD flow with non-ideal boundaries, Commun. Appl. Anal. 3 (1999) 383-398.

[36] A. J. Meir, P. G. Schmidt, Analysis and finite element simulation of mhd flows, with an application to liquid metal processing, in: Fluid Flow Phenomena in Metals Processing, 1999, pp. 561-569. 
[37] A. J. Meir, P. G. Schmidt, Analysis and numerical approximation of a stationary mhd flow problem with nonideal boundary, SIAM J. Numer. Anal. 34 (1999) 1304-1332.

[38] A. J. Meir, P. G. Schmidt, S. I. Bakhtiyarov, R. A. Overfelt, Numerical simulation of steady liquid metal flow in the presence of a static magnetic field, J. Appl. Mech. 71 (6) (2004) 786-795.

[39] M. Xu, F. Stefani, G. Gerbeth, The integral equation method for a steady kinematic dynamo problem, J. Comput. Phys. 196 (1) (2004) 102-125. doi:10.1016/j.jcp.2003.10.034.

[40] M. Xu, F. Stefani, G. Gerbeth, Integral equation approach to timedependent kinematic dynamos in finite domains, Phys. Rev. E 70 (5) (2004) 102-125. doi:10.1103/PhysRevE.70.056305

[41] M. Xu, F. Stefani, G. Gerbeth, The integral equation approach to kinematic dynamo theory and its application to dynamo experiments in cylindrical geometry, J. Comput. Phys. 227 (17) (2008) 8130-8144. doi:10.1016/j. jcp.2008.05.009.

[42] N. Weber, V. Galindo, F. Stefani, T. Weier, The Tayler instability at low magnetic Prandtl numbers: Between chiral symmetry breaking and helicity oscillations, New J. Phys. 17 (11) (2015) 113013. doi:10.1088/ $1367-2630 / 17 / 11 / 113013$.

[43] F. Stefani, A. Giesecke, N. Weber, T. Weier, Synchronized Helicity Oscillations: A Link Between Planetary Tides and the Solar Cycle?, Sol. Phys. 291 (8) (2016) 2197-2212. doi:10.1007/s11207-016-0968-0.

[44] L. A. Santalo, Vectores y Tensores Con Sus Aplicaciones, Universidad de Buenos Aires, 1993.

[45] N. Weber, P. Beckstein, V. Galindo, W. Herreman, C. Nore, F. Stefani, T. Weier, Metal pad roll instability in liquid metal batteries, Magnetohydrodynamics 53 (1) (2017) 129-140. 
[46] N. Weber, P. Beckstein, W. Herreman, G. M. Horstmann, C. Nore, F. Stefani, T. Weier, Sloshing instability and electrolyte layer rupture in liquid metal batteries, Phys. Fluids 29 (5) (2017) 054101. doi:10.1063/1. 4982900

[47] R. Moreau, Magnetohydrodynamics, Kluwer Academic Publishers, 1990.

[48] J. U. Brackbill, D. C. Barnes, The Effect of Nonzero $\nabla \cdot$ B on the numerical solution of the magnetohydrodynamic equations, J. Comput. Phys. 35 (1980) 426-430.

[49] T. Miyoshi, K. Kusano, A Comparative Study of Divergence-Cleaning Techniques for Multi-Dimensional MHD Schemes, Plasma Fusion Res. 6 (2011) 2401124. doi:10.1585/pfr.6.2401124.

[50] O. Bíró, K. Preis, On the use of the magnetic vector potential in the finite element analysis of three-dimensional eddy currents, IEEE Transactions on Magnetics 25 (4) (1989) 3145-3159. doi:10.1109/20.106343

[51] C. Greenshields, OpenFOAM Programmer's Guide, OpenFOAM Foundation, 2012.

[52] J. K. Carson, S. J. Lovatt, D. J. Tanner, A. C. Cleland, Thermal conductivity bounds for isotropic, porous materials, Int. J. Heat Mass Transf. 48 (11) (2005) 2150-2158. doi:10.1016/j.ijheatmasstransfer.2004.12.032.

[53] S. S. Kumar, Y. M. C. Delaur, Convective Heat Transfer of an Air Bubble in Water With Variable Thermophysical Properties, Heat Transf. Eng. 35 (2014) 1370-1379. doi:10.1080/01457632.2013.877317.

[54] H. Marschall, K. Hinterberger, C. Schler, F. Habla, O. Hinrichsen, Numerical simulation of species transfer across fluid interfaces in free-surface flows using OpenFOAM, Chem. Eng. Sci. 78 (2012) 111-127. doi:10.1016/j. ces.2012.02.034. 
[55] M. Nabil, A. S. Rattner, interThermalPhaseChangeFoam-A framework for two-phase flow simulations with thermally driven phase change, SoftwareX 5 (2016) 216-226. doi:10.1016/j.softx.2016.10.002

[56] R. I. Issa, Solution of the Implicit Discretised Fluid Flow Equations by Operator-Splitting, J. Comput. Phys. 62 (1985) 40-65.

[57] J. H. Wilkinson, The Algebraic Eigenvalue Problem, Oxford University Press, 1965.

[58] B. N. Parlett, The Symmetric Eigenvalue Problem, Society for Industrial and Applied Mathematics, 1998. doi:10.1137/1.9781611971163

[59] Y. Saad, Numerical Methods for Large Eigenvalue Problems, rev. ed Edition, no. 66 in Classics in applied mathematics, Society for Industrial and Applied Mathematics, 2011.

[60] R. A. Woods, D. R. Milner, Motion in the weld pool in arc welding, Weld. J. 50 (1971) 163-173.

[61] A. I. Dementev, A. I. Chaikovskii, A. Y. Chudnovskii, Generation of electrovortex flows in liquid-metal baths with a multielectrode current input, Magnetohydrodynamics 24 (1) (1988) 76-80.

[62] S. B. Dement'ev, O. M. Skopis, E. V. Sherbinin, Intensification of the mixing process in direct-current electric arc furnaces, Magnetohydrodynamics 28 (1) (1992) 89-92.

[63] I. E. Butsenieks, D. E. Peterson, V. I. Sharamkin, E. V. Sherbinin, Magnetohydrodynamic fluid flows in a closed space with a nonuniform electric current, Magn. Gidrodin. 1 (1976) 92-97.

[64] R. P. Millere, V. I. Sharamkin, E. V. Shcherbinin, Effect of a longitudinal magnetic field on electrically driven rotational flow in a cylindrical vessel, Magn. Gidrodin. 1 (1980) 81-85. 
[65] V. N. Moshnyaga, V. I. Sharamkin, Experimental investigation of electrovortical flow in a cylindrical vessel, Magnetohydrodynamics 16 (1) (1980) $62-65$.

[66] L. A. Volokhonskii, Dynamic boundary layer of electrovortex flow in a cylindrical volume with axisymmetric current supply, Magnetohydrodynamics 27 (4) (1991) 467-470.

[67] A. Y. Chudnovskii, Evaluating the intensity of a single class of electrovortex flows MHD, Magnetohydrodynamics 25 (3) (1989) 406-408.

[68] V. G. Zhilin, Y. P. Ivochkin, A. A. Oksman, G. R. Lurin'sh, A. I. Chaikovskii, A. Y. Chudnovskii, E. V. Shcherbinin, An experimental investigation of the velocity field in an axisymmetric electrovortical flow in a cylindrical container, Magn. Gidrodin. 3 (1986) 110-116.

[69] International Atomic Energy Agency, Thermophysical Properties of Materials for Nuclear Engineering: A Tutorial and Collection of Data., International Atomic Energy Agency, 2008.

[70] H. Bettin, H. Fehlauer, Density of mercury - measurements and reference values, Metrologia 41 (2004) 16-23.

[71] H. Kim, D. A. Boysen, J. M. Newhouse, B. L. Spatocco, B. Chung, P. J. Burke, D. J. Bradwell, K. Jiang, A. A. Tomaszowska, K. Wang, W. Wei, L. A. Ortiz, S. A. Barriga, S. M. Poizeau, D. R. Sadoway, Liquid Metal Batteries: Past, Present, and Future, Chem. Rev. 113 (3) (2013) 2075-2099. doi:10.1021/cr300205k.

[72] R. Ashour, D. H. Kelley, A. Salas, M. Starace, N. Weber, T. Weier, Competing forces in liquid metal electrodes and batteries, J. Power Sources 378 (2018) 301-310. doi:10.1016/j.jpowsour.2017.12.042.

[73] J. W. Evans, D. P. Ziegler, The Electrolytic Production of Aluminum, in: A. Bard, M. Startmann (Eds.), Electrochemical Engineering, Vol. 5 of 
Encyclopedia of Electrochemistry, Wiley-VCH, 2007, pp. 224-265, volume editors: Macdonald, D.D. and Schmuki, P.

[74] M. Starace, N. Weber, M. Seilmayer, C. Kasprzyk, T. Weier, F. Stefani, S. Eckert, Ultrasound Doppler flow measurements in a liquid metal column under the influence of a strong axial electric current, Magnetohydrodynamics 51 (2015) 249-256.

[75] D. H. Kelley, D. R. Sadoway, Mixing in a liquid metal electrode, Phys. Fluids 26 (5) (2014) 057102. doi:10.1063/1.4875815

\section{Appendix A. Detailed simulation of test case 3}

In this section we present further details of test case 3. Figure A.9 shows the grid study: at a current of $200 \mathrm{~A}, 60$ cells on the diameter show convergence of the jet velocity. Figure A.10 shows the steady state jet velocity at $200 \mathrm{~A}$ using an old segregated [11] and the here developed solver. The results match perfectly; the coupled solver was more than 10 times faster than the segregated one.

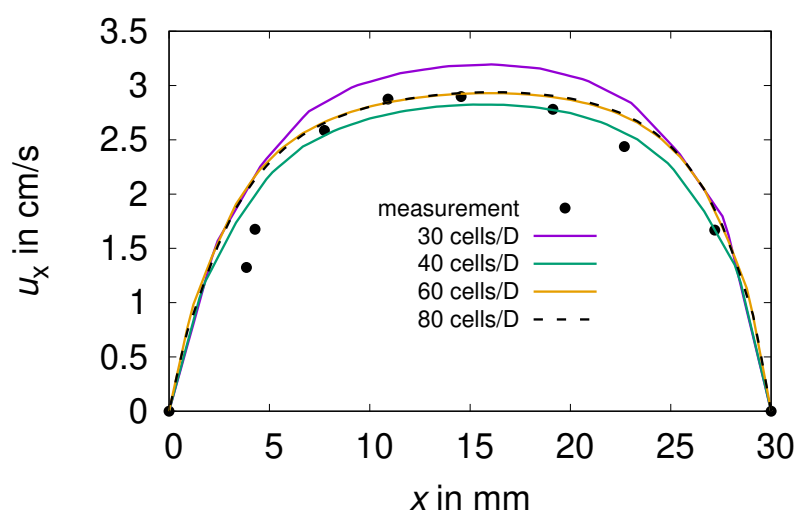

Figure A.9: Grid study for $I=200$ A. The mesh is refined between 30 and 80 cells on the diameter; only cubic cells are used. The curves show the steady state velocity along the jet.

Finally, we illustrate in figure A.11 the jet velocity at $200 \mathrm{~A}$ for a variation of the copper and steel electrical conductivity and the length of the rod. These 


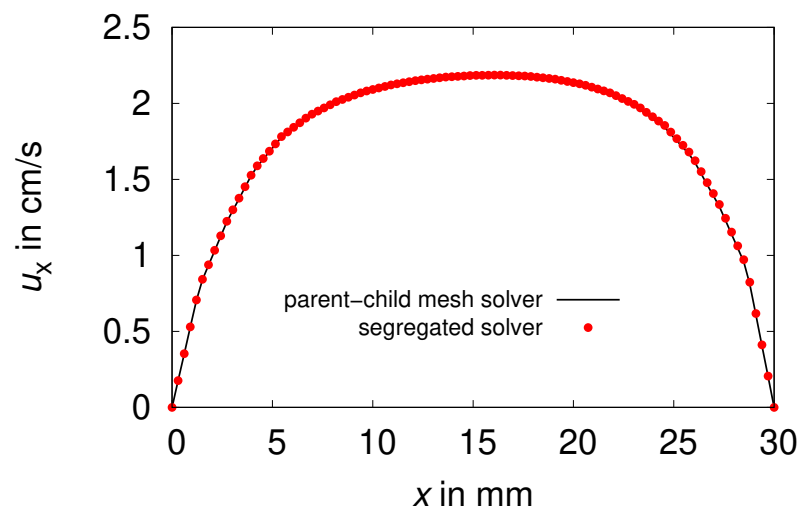

Figure A.10: Comparison of the segregated (see [11]) and parent-child mesh solver. The current is $I=200 \mathrm{~A}, 50$ cells/diameter are used.

properties / measurements are not exactly known from the experiment. While the exact conductivity of the copper conductors is negligible, the conductivity of the steel tube and the length of the rod change the steady state velocity. We illustrate further the influence of the induced current and the Earth magnetic field. We see, that the induced current and magnetic field are negligible. However, the (vertical) Earth magnetic field changes not only the speed of the jet, but also its shape. We suspect, that other vertical magnetic fields (from the feeding lines) may have an additional influence on the jet. 


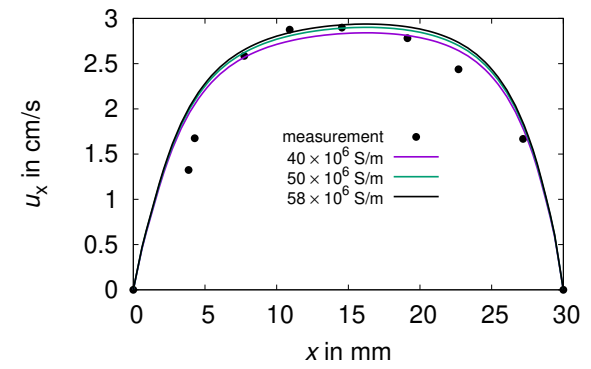

(a)

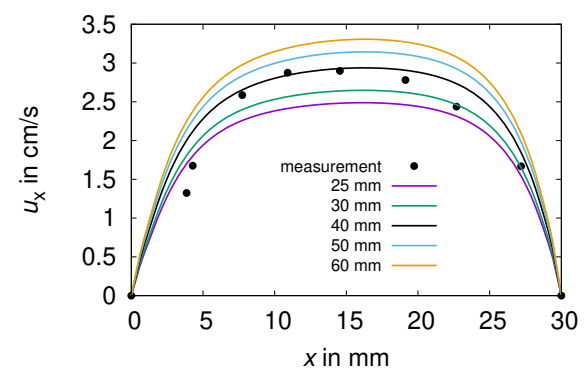

(c)

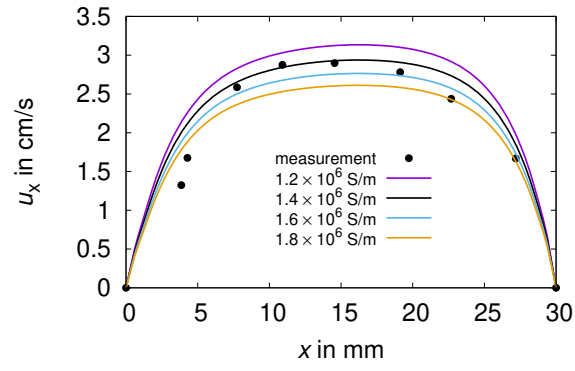

(b)

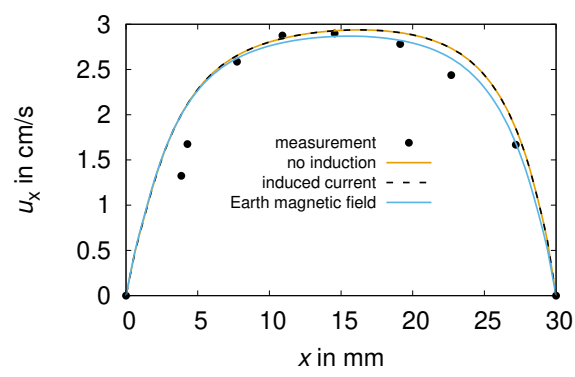

(d)

Figure A.11: Steady state flow velocity along the jet for $I=200$ A. In (a) the conductivity of the copper electrodes is varied, in (b) the conductivity of the steel tube. A variation of the length of the rod (c) has a significant influence on the flow speed. Finally, (d) illustrates the influence of the Earth magnetic field and the induced current. 